Pacific Journal of Mathematics

SOME MAPPING PROPERTIES OF THE GROUP ALGEBRAS 


\title{
SOME MAPPING PROPERTIES OF THE GROUP ALGEBRAS OF A COMPACT GROUP
}

\author{
Charles A. AKemann
}

\begin{abstract}
A number of equivalent conditions are given under which certain representations of $M(G)$ and $L^{1}(G)$ have closed range. A faithful representation with closed range implies the finiteness of $G$. Weakly compact operators on $L^{1}(G)$ commuting with right translations are classified as left convolutions by functions in $L^{1}(G)$.
\end{abstract}

In recent years various authors have shown that the same techniques which have proved so successful in developing the theory of locally compact abelian groups can be applied, with surprisingly small modifications, to nonabelian groups as well. The purpose of this paper is to carry on this theme with special emphasis on compact groups. Toward this end we shall emphasize the use of functional analysis methods and representation theory. In fact, the main technical result of the paper doesn't concern groups at all, though its foremost application, Theorem 2, gives some equivalent conditions under which certain representations of the group algebras of a compact group have closed range. The other main result of the paper is Theorem 4, which characterizes the weakly compact operators in the group algebra $L^{1}(G)$ [ $G$ compact] which commute with right translations as left convolution by some element of $L^{1}(G)$.

1. Notation and preliminaries. Let $G$ be a compact Hausdorff group. Following Dixmier [2] we denote the set of equivalence classes of irreducible unitary representations of $G$ by $\widehat{G}$. We shall assume that one representation is chosen from each equivalence class, and we shall denote this collection by $\widehat{G}$ as well. If $U_{r}$ is in $\hat{G}$, then by the Peter-Weyl Theorem, $U_{r}$ is a finite-dimensional representation, say of dimension $d_{r}$, on the Hilbert space $H_{r}$. For each such $r$ let $B\left(H_{r}\right.$.) be the full matrix algebra over $H_{r}$ with the usual operator norm. Let $T C\left(H_{r}\right)$ be the full matrix algebra over $H_{r}$ with the trace norm [i.e. the trace class of $H_{r}$ ]. Let $M$ be the bounded direct product of all the $B\left(H_{r}\right)$. That is, if $\Delta$ is an index set for $\hat{G}$, then $M$ is that subset of the Cartesian product of $\left\{B\left(H_{r}\right)\right\}_{r \in \Delta}$ for which $\left\{\left\|a_{r}\right\|\right\}_{r \in \Delta}$ is bounded, where $\left\{a_{r}\right\} \in M$. Under coordinatewise operations and norm $\|a\|=\sup _{r \in \Delta}\left\|a_{r}\right\|$, for $a=\left\{a_{r}\right\} \in M$, it is well-known that $M$ is a $W^{*}$-algebra, i.e. a von Neumann algebra. Let $N$ be the subalgebra of $M$ defined by the condition: $a \in N$ if and only if for any $\alpha>0,\left\{r \in \Delta:\left\|a_{r}\right\|>\alpha\right\}$ is a finite set. It is clear that $N$ is a norm-closed subalgebra of $M$ and hence a $B^{*}$-algebra. Let $F$ 
be the Banach space direct sum of the $\left\{T C\left(H_{r}\right)\right\}_{r \in \Delta}$, i.e. if $c=\left\{c_{r}\right\} \in F$, $\|c\|=\sum_{r \in \Delta}\left\|c_{r}\right\|_{T}$. It is easy to verify that as Banach space, $F$ is the dual of $N$ [written $F=N^{*}$ ] and $M$ is the dual of $F$ under the bilinear mapping $\operatorname{tr}(a c)$ for $a \in M$ or $N$ and $c \in F . \quad\left[\operatorname{tr}(a c)=\sum_{r \in \Delta} \operatorname{tr}\left(a_{r} c_{r}\right)\right]$. We define a representation $U$ of $G$ into $M$ by $U(t)=\left\{U_{r}(t)\right\}$.

We define the $L^{p}$ spaces for $G$ with respect to Haar measure in the usual way. Also we shall consider the space $C(G)$ of continuous complex-valued functions on $G$ with sup norm, and its dual, the space $M(G)$ of finite regular Borel measures on $G$. It is well-known that both $M(G)$ and $L^{1}(G)$ are Banach *algebras under convolution multiplication. Also $L^{1}(G)$ is naturally embedded in $M(G)$ as a closed ideal which is dense in the weak* topology of $M(G)$. When it is convenient we shall identify $L^{1}(G)$ with its natural embedding in $M(G)$, and we shall do the same with $N$ and its embedding in $M$.

We now define the Fourier transform as an $M$-valued *homomorphism of $M(G)$. If $m$ is in $M(G)$, define the Fourier transform $\Lambda(m) \stackrel{d f}{=} \hat{m}$ to be the operator in $M$ with $r^{\text {th }}$-component $\int_{\theta} U_{r}(t) d m(t)$. The fact that $\Lambda$ is an injective *homomorphism of $M(G)^{*}$ into $M$ is proved in Dixmier [2, p. 316]. Dixmier also proves that if $\Lambda$ is restricted to $L^{1}(G)$, then the range of $A$ lies in $N$. Thus $A$ induces a dual map $A^{*}$ of $F$ into $L^{\infty}(G)$. If $c$ is in $F$ and $f$ is in $L^{1}(G)$, then

$$
A^{*}(c)(f)=c(\hat{f})=\operatorname{tr}\left(c\left(\int_{\theta} U(t) f(t) d t\right)\right)=\int_{\theta} f(t) \operatorname{tr}(c U(t)) d t .
$$

This proves that $\Lambda^{*}(c)(t)=\operatorname{tr}(c U(t))$, and hence the range of $\Lambda^{*}$ actually lies in the natural embedding of $C(G)$ in $L^{\infty}(G)$. Thus we may consider $\Lambda^{* *}$, which maps $L^{\infty}(G)^{*}$ into $M$, as actually mapping $M(G)$ into $M$ by composing $\Lambda$ with the natural quotient map of $L^{\infty}(G)^{*}$ onto $M(G)$. Thus $A$ is a dual map and almost a second dual map, and we shall tend to identify the various maps $\Lambda$ used above when no confusion can result.

Finally we note that if $p$ is any central projection in $M$, then the spaces $p N, p F$, and $p M$ are defined in the obvious way, and we have immediately that $(p N)^{*}=p F$ and $(p F)^{*}=p M$. We shall consider $p M$ as a subalgebra of $M, p F$ as a subspace of $F$, and $p N$ as a subalgebra of $N$.

Proposition A. $N$ is the $C^{*}$ group algebra of $G$, and $M$ is the "big group algebra of $G$ ". [cf. 4]

Proof. We first show that $\hat{L}^{1}(G)$ is uniformly dense in $N$. In order to show this it is only necessary to point out that if $p$ is the central projection in $N$ determined by the finite set $U_{r_{1}}, \cdots, U_{r_{n}}$ in $G$, then $p=\left(d_{r_{1}} \chi_{r_{1}}+\cdots+d_{r_{n}} \chi_{r_{n}}\right)^{\wedge} \cdot\left[\chi_{r}(t)=\operatorname{tr}\left(U_{r}(t)\right)\right]$. This proves that 
$p N=p \hat{L}^{1}(G)$, and hence $\hat{L}^{1}(G)$ is uniformly dense in $N$. Also if $A$ is in $\hat{L}^{1}(G)$, then $C^{*}$ norm of $A$ equals the norm (as an element of $N$ ) of $\hat{A}$ by [2, p. 40]. Thus $N$ is naturally isomorphic to the abstract completion of $L^{1}(G)$ in the $C^{*}$ norm, so $N$ is the $C^{*}$ group algebra of $G$. It is then immediate from [4, p. 476] that $M$ is the "big group algebra of $G$ ".

The above result identifies the objects of the present study as the familiar group algebras. The ideal structure of $M$ and $N$ is particularly simple, and it follows easily that the ideal structure of $L^{1}(G)$ is essentially the same as that of $N$.

Proposition B. The closed ideals of $L^{1}(G)$ are precisely those of the form $\{f: p \hat{f}=0\}$ for some central projection $p$ of $M$. The weak* closed ideals of $M(G)$ are precisely those of the form $\{m: p \hat{m}=0\}$ for some central projection $p$ of $M$.

Proof. Loomis [11, p. 161] proves the assertion about $L^{1}(G)$ though in slightly differenty form. Alternately one may use the methods of Rudin [12]. To prove the assertion about $M(G)$ by these methods one needs only to note that if $\left\{f_{\alpha}\right\}$ is a bounded approximate identity in $L^{1}(G)$, then $\left\{f_{\alpha}\right\}$ converges to $I$ in the weak* topology of $M$. Otherwise one replaces characters with those functions determined by taking a specific matrix representation for each $U_{r}$ and looking at each entry. Compare [9] for more details of this type of substitution.

We remark here that the most important aspect of the structure we are building here is that one can use the methods of the abelian case to prove results analogous to the familiar ones. For example we could imitate again the proof in [12] to prove the following characterization of $\hat{M}(G)$ as a subset of $M$.

Proposition C. Suppose $a \in M$. Then $a \in \hat{M}(G)$ if and only if for each $f \in L^{1}(G)$, $a \hat{f} \in \hat{M}(G)$.

2. Representation of $L^{1}(G)$ and $M(G)$. For the first result of this section we suspend our notation temporarily in order to more readily state Theorem 1 in its full generality. This theorem was first proved by W. G. Bade [unpublished] for the abelian case, and, as usual, our proof draws heavily on the original methods.

Theorem 1. Let $\Omega$ be any index set and suppose that for each $\theta$ in $\Omega$ we are given a finite dimensional Hilbert space $H_{\theta}$. Let $H$ be the direct sum of the $\left\{H_{\theta}\right\}_{\theta \in \Omega}, M$ be the bounded direct product of the $\left\{B\left(H_{\theta}\right)\right\}_{\theta \in \Omega}$, and $F$ be the direct sum of the $\left\{T C\left(H_{\theta}\right)\right\}_{\theta \in \Omega}$. Then 
$F^{*}=M$ and $M$ is a $W^{*}$-algebra acting on $H$ in the natural way. Suppose $X$ is a Banach space and $T$ is a bounded linear operator mapping $F$ into $X$. Suppose further that for each unitary operator $u$ in $M$ there exists $x^{*}$ in $X^{*}$ such that $\left\|u-T^{*}\left(x^{*}\right)\right\|<1$. Then $T^{*}$ is surjective.

Proof. Let $U$ be the set of unitary operators of $M$. We claim that $U$ is closed in the weak operator topology (equivalently the $\sigma(M, F)$ topology) of $M$. To see this suppose $\left\{u_{\varphi}\right\}$ is a net in $U$ which $\sigma(M, F)$ converges to some $a$ in $M$. Because of the way that $M$ acts on $H$ we need only show that $a$ is unitary on each $H$, . But each $H_{\text {, }}$ is finite dimensional, so $\left\{u_{\varphi}\right\}$ converges uniformly to $a$ on each $H_{\theta}$, so $a$ is unitary on each $H_{\theta}$, so $a$ is in $U$. Thus $U$ is compact for the $\sigma(M, F)$ topology of $M$.

We need only show that $T$ is injective and has closed range, whence it follows that $T^{*}$ is surjective. Suppose $T(f)=0$ for some $f \in F$. Suppose $\|f\|=1$. By [13] and the above $\exists$ unitary $u \in M \ni f(u)=1$. Choose $x^{*} \in X^{*} \ni\left\|T^{*}\left(x^{*}\right)-u\right\|<1$. Then

$$
1>|| T^{*}\left(x^{*}\right)-u \| \geqq\left|f\left(T^{*}\left(x^{*}\right)\right)-f(u)\right|=|f(u)|=1 .
$$

This contradiction shows $T$ is injective.

To show $T$ has closed range we need only find a finite dimensional central projection $p \in M \ni$ the restriction of $T$ to $(I-p) F$ has closed range, since $F=p F \oplus(I-p) F$ and $p F$ is finite dimensional. Assuming no such $p$ exists, we may find by induction sequences $\left\{f_{N}\right\}$ in $F$ and $\left\{p_{N}\right\}$ orthogonal, finite-dimensional, central projections satisfying: $\left\|f_{N}\right\|=1, f_{N}(a)=f_{N}\left(p_{N} a\right) \forall a \in M,\left\|T\left(f_{N}\right)\right\| \rightarrow 0$. By [13] and the orthogonality of the $\left\{p_{N}\right\} \quad \exists u$ unitary in $M \ni f_{N}(u)=1$ for all $N=$ $1,2, \cdots$. Choose $x^{*} \in X^{*} \ni\left\|T^{*}\left(x^{*}\right)-u\right\|<1$. Then

$$
\begin{aligned}
1=\left\|f_{N}\right\| & =f_{N}(u) \leqq\left|f_{N}\left(u-T^{*}\left(2^{*}\right)\right)\right|+\left|f_{N}\left(T^{*}\left(x^{*}\right)\right)\right| \\
& \leqq\left\|u-T^{*}\left(x^{*}\right)\right\|+\left\|T\left(f_{N}\right)\right\| \cdot\left\|x^{*}\right\| \cdot
\end{aligned}
$$

Letting $N$ tend to $\infty$ we get $1 \leqq\left\|u-T^{*}\left(x^{*}\right)\right\|$, a contradiction.

For the case of an arbitrary $W^{*}$-algebra the conclusion of the last theorem will in general be false. We shall give an example to illustrate this.

ExAMPle D. Let $H$ be a separable infinite dimensional Hilbert space and set $M=B(H)$ and $F=T C(H)$. Let $\left\{x_{n}\right\}$ be a fixed orthonormal basis in $H$, and we shall write elements of $F$ and $M$ as matrices with respect to this basis. First note that $F$ is continuously isomorphic to the Banach space $l_{2}+F$ under the mapping: $\theta\left(\left\{f_{i j}\right\}\right)=$ 
$\left\{f_{1 j}\right\}+\left\{f_{i+1, j}\right\}$. Set $Y=l_{2}+F$, then $Y^{*}$ is isomorphic to the direct product of $l_{2}$ and $M$ (written $l_{2} \pi M$ ). Also $\theta^{*}$ takes $Y^{*}$ into $M$ by: $\theta^{*}\left(\left\{a_{i 0}\right\}+\left\{a_{i j}\right\}\right)=\left\{a_{i, j-1}\right\}$, as is easily checked by considering matrices.

Now let $X=c_{0}+F$, and consider the map $T$ of $Y$ into $X$ defined by $T\left(\left\{f_{n}\right\}+\left\{f_{i j}\right\}\right)=\left\{f_{n}\right\}+\left\{f_{i j}\right\}$. It is immediate that $T$ is continuous and that $T^{*}\left(X^{*}\right)$ has dense range in $Y^{*}$. (One checks that

$$
T^{*}\left(\left\{e_{n}\right\}+\left\{a_{i j}\right\}\right)=\left\{e_{n}\right\}+\left\{a_{i j}\right\},
$$

where $\left\{e_{n}\right\}$ is in $l_{1}$, and we are considering $X^{*}$ as isomorphic to $l_{1} \pi M$.) However, $T^{*}$ is not surjective since $l_{1}$ is a proper subset of $l_{2}$.

We are almost ready to state and prove the main theorem of this section. In the abelian case this result is proved in [12, Ch. 5], and again many of our methods are similar. Another approach to the nonabelian case is given in [9], but their results are not equivalent to ours unless the group $G$ admits only irreducible representations of bounded order. A discussion of such groups is given by Kaplansky [10]. The subject also arose in [6], and some of the equivalences of Theorem 2 of the present paper are proved therein, though they are stated in different form. For notational convenience we first introduce some definitions.

DEFINITION E. Choosing in any fashion orthonormal bases in the $H_{r}$ we may write, for each $t \in G, U_{r}(t)$ as a matrix $U_{r}^{i j}(t)$. For each fixed triple $(r, i, j)$ the function $U_{r}^{i j}(t)$ is well-defined on $G$, and we call a function $f$ on $G$ a polynomial or trigonometric polynomial if it is a finite linear combination of such functions.

Definition F. Let $E$ be a subset of $\Delta$, the index set of $\hat{G}$. The central projection of $M$ corresponding to $E$ is that unique projection $p$ such that $p M=M_{E}$, where $M_{E}$ is the bounded direct product of the algebras $\left\{B\left(H_{r}\right)\right\}_{r \in E}$ considered as a subalgebra of $M$ in the natural way. $f \in L^{1}(G)$ is called an E-function if $p \hat{f}=\hat{f}$ ( $p$ as above). A polynomial $f$ is called an E-polynomial if $p \hat{f}=\hat{f}$.

THEOREM 2. Let $E$ be a subset of $\triangle$ and $p$ the central projection of $M$ corresponding to $E$. Then the following statements are equivalent:

(0) Definition: $E$ is a Sidon set.

(1) $p \widehat{M}(G)=p M$.

(2) $p \hat{L}^{1}(G)=p N$.

(3) $\Lambda^{*}$ restricted to $p F$ has closed range.

(4) If $f$ is a continuous E-function, then $\sum_{r \in E} d_{r} \operatorname{tr}\left(\left|\hat{f}_{r}\right|\right)<\infty$.

(5) If $f$ is a bounded E-function, then $\sum_{r \in E} d_{r} \operatorname{tr}\left(\left|\hat{f}_{r}\right|\right)<\infty$. 
(6) There exists $K>0$ such that if $f$ is an E-polynomial, then $K\|f\|_{\infty} \geqq \sum_{r \in E} d_{r} \operatorname{tr}\left(\left|\hat{f}_{r}\right|\right)$.

(7) There exists $K>0$ such that if $f$ is a bounded E-function, then $K\|f\|_{\infty} \geqq \sum_{r \in E} d_{r} \operatorname{tr}\left(\left|\hat{f}_{r}\right|\right)$.

(8) For each unitary $u$ in $M$ there exists $m \in M(G)$ such that $\|p(u-\hat{m})\|<1$.

Proof. The equivalence of (1)-(3) follows from [3, pp. 487-488]. The implications $(7) \rightarrow(6),(7) \rightarrow(5),(5) \rightarrow(4)$ are obvious. Theorem 1 and the previous remark that $\Lambda$ is a dual map shows the equivalence of (1) and (8). We now fill in the gaps.

$(4) \rightarrow(3)$ : If $f$ is in the closure of the range of $\Lambda^{*}$, it is immediate that $f$ is a continuous $E$-function. Thus by the hypothesis (4), $c=\left\{d_{r} f_{r}\right\}$ is a well-defined element of $F$. One easily verifies that $\Lambda^{*}(c)=f$. Thus (3) holds.

$(3) \rightarrow(6):$ By $(3)$ and the closed graph theorem we need only to note that any $E$-polynomial $f$ trivially satisfies $\sum_{r \in E} d_{r} \operatorname{tr}\left(\left|\hat{f}_{r}\right|\right)<\infty$. Thus $f$ is in the range of $A^{*}$ as above, and (6) follows by the closed graph theorem.

$(6) \rightarrow(7)$ : Let $f$ be a bounded $E$-function, and let $\alpha>0$ be given. Fix $r_{1}, \cdots, r_{n}$ in $E$. Since there exists a bounded approximate identity consisting of trigonometric polynomials, we may choose such a polynomial $g$ such that $\sum_{i=1}^{N} d_{r_{i}} \operatorname{tr}\left(\left|\hat{f}_{r_{i}}\right|\right)-\alpha \leqq \sum_{i=1}^{N} d_{r_{i}} \operatorname{tr}\left(\left|\hat{f}_{r_{i}} \hat{g}_{r_{i}}\right|\right) \leqq$ $K\||f| *|g|\|_{\infty} \leqq K\|f\|_{\infty}\|g\|_{1}$ and $\|g\|_{1} \leqq 1$, where $K$ is the constant given by (6). This proves (7), and the theorem follows.

REMARK. A useful example of a set $E$ satisfying the conditions of the last theorem is given in [6]. A related concept is discussed in $[8]$.

It is to be noted here that sets $E$ which satisfy the conditions of Theorem 2 will have to be sparse in some sense. It is known that in the abelian case no representation of $L^{1}(G)$ or $M(G)$ can both be faithful and have closed range. With the tools at hand nowadays it is easy to extend this result to the general case-even for noncompact $G$.

THEOREM 3. Let $G$ be any locally compact group and suppose $T: M(G)\left[L^{1}(G)\right] \rightarrow B(H)$, for some Hilbert space $H$, is faithful. If $A$ is a $C^{*}$-algebra in $B(H)$ and $A$ is a subset of the range of $T$, then $A$ is finite dimensional.

Proof. Let $J=T^{-1}(A)$. Since $T$ is norm-decreasing [2], $J$ is a closed subspace of $M(G)$. Thus $J$ is weakly sequentially complete. By the closed graph theorem, $T$ is a homeomorphism on $J$, so $A$ is weakly 
sequentially complete as well. But Sakai [14] proves that such an $A$ is finite-dimensional.

3. Operators in $L^{1}(G)$. The earliest results of the type to be given here were proved by Wendel [16] who showed that a bounded linear operator $T: L^{1}(G) \rightarrow L^{1}(G)$ which commuted with right translations was just left convolution by some $m \in M(G)$. Helgason [8] used this fact to classify such operators $T$ (as above) which were also spectrally continuous as convolutions by functions in $L^{2}(G)$. Sakai [15] eliminated some unnecessary hypotheses from Helgason's theorem. We shall use the results of all of these papers for our classification theorem.

THEOREm 4. Suppose $T: L^{1}(G) \rightarrow L^{1}(G)$ is a bounded linear map which commutes with right translations. Then the following conditions on $T$ are equivalent:

(1) $T$ is a compact operator.

(2) $T$ is a weakly compact operator.

(3) There exists $g \in L^{1}(G)$ such that for each $f \in L^{1}(G), T(f)=$ $g * f$.

Proof. Wendel [16] proves that $T$ is just left convolution by some $m \in M(G)$ and $\|m\|=\|T\|$. Clearly (1) implies (2). Also it follows by standard arguments that the space of compact [weakly compact] operators on $L^{1}(G)$ of this type is closed in the norm topology of $B\left(L^{1}(G)\right.$ ). If $g \in L^{2}(G)$, the map $f \rightarrow g * f$ is spectrally continuous by a theorem of Helgason [9]. That is to say, it can be extended to a continuous linear map of $N$ into $L^{1}(G)$; and hence it is weakly compact by a result of [15]. Since $L^{2}(G)$ is dense in $L^{1}(G)$, it follows that (3) implies (2). By [3, p. 510] if $T$ and $T^{\prime}$ are weakly compact operators on $L^{1}(G)$, then their composition $T T^{\prime}$ is compact. Since $L^{1}(G) * L^{1}(G)$ is dense in $L^{1}(G)$, it follows that (3) implies (1) as well.

To complete the proof we assume that $m$ (as above) is not absolutely continuous and show that this implies $T$ is not weakly compact. By considering the Jordan decomposition of $m$ and using the EberleinSmulian Theorem [3, p. 430] we may assume that $m$ is positive. Since $m$ is not absolutely continuous, there exists a compact subset $E$ of $G$ such that $E$ is a null set for the Haar measure of $G$ but $m(E)>0$. Let $\theta$ be the Haar measure of $G$. Choose open neighborhoods $\left\{V_{n}\right\}$ of the identity $e$ of $G$ and neighborhoods $\left\{U_{n}\right\}$ of $E$ such that $\theta\left(U_{n}\right) \rightarrow 0$ and $E V_{n} \subset U_{n}$. Let $f_{n}$ be defined by $f_{n}(t)=1 / \theta\left(V_{n}\right)$ if $t \in V_{n}$ and $f_{n}(t)=0$ otherwise. Then $\left\|f_{n}\right\|_{1}=1$ for each $n=1,2, \ldots$. Also we have $\int_{E V_{n}} m * f_{n}(t) d t=\int_{\theta}\left[\int_{E V_{n}} f_{n}\left(s^{-1} t\right) d t\right] d m(s)$. But for each $s \in E$, 


$$
\int_{E V n} f_{n}\left(s^{-1} t\right) d t \geqq \int_{s V_{n}} f_{n}\left(s^{-1} t\right) d t=\int_{V_{n}} f_{n}(t) d t=1
$$

Thus we have,

$$
\int_{E V_{n}} m * f_{n}(t) d t \geqq \int_{E} 1 d m \geqq m(E), \quad \text { for each } n=1,2, \cdots \text {. }
$$

But $\theta\left(E V_{n}\right) \leqq \theta\left(U_{n}\right) \rightarrow 0$. By [3, p. 294] this shows that $\left\{m * f_{n}\right\}$ is not a weakly relatively compact set and hence $T$ is not a weakly compact map.

We remark that the argument of Sakai [15] show that if $G$ is not compact (but is locally compact), then any map $T$ satisfying the conditions of the last Theorem is identically 0 .

\section{BIBLIOGRAPHY}

1. W. G. Bade and P. C. Curtis, Jr., Embedding theorems for commutative Banach algebras (to appear in Pacific J. Math.).

2. J. Dixmier, Les $C^{*}$-algebras et leurs representations, Paris, 1964.

3. N. Dunford and J. Schwartz, Linear Operators, Part I: General Theory, New York, 1958.

4. J. Ernest, $A$ new group algebra for locally compact groups, Amer. J. Math. 87 (1964), 467-492.

5. L. Ernest, $A$ new group algebra for locally compact groups II, Canad. J. Math. 17 (1965), 604-615.

6. A. Figa-Talamanca and D. Rider, $A$ theorem of Littlewood and lacunary series for compact groups, Pacific J. Math. 16 (1966), 505-514.

7. - A theorem on random Fourier series on noncommutative groups (to appear).

8. S. Helgason, Topologies of group algebras and a theorem of Littlewood, Proc. Amer. Math. Soc. 9 (1958), 782-790.

9. - Topologies of group algebras and a theorem of Littlewood, Trans. Amer. Math. Soc. 86 (1957), 269-283.

10. I. Kaplansky, Groups with representations of bounded order, Canad. J. Math. 1 (1949), 105-112.

11. L. Loomis, An Introduction to Abstract Harmonic Analysis, New York, 1953.

12. W. Rudin, Fourier Analysis on Groups, New York, 1962.

13. B. Russo and H. Dye, A note on unitary operators in $C^{*}$-algebras (to appear in Duke Math. J.).

14. S. Sakai, The theory of $W^{*}$-algebras, Yale Notes, 1962.

15. - Weakly compact operators on operator algebras, Pacific J. Math. 14 (1964), 659-664.

16. J. Wendel, Left centralizers and isomorphisms of group algebras, Pacific J. Math. 2 (1952), 251-261.

Received July 28, 1966.

University of California, Berkeley and University of PenNsylvania 


\section{PACIFIC JOURNAL OF MATHEMATICS}

\section{EDITORS}

\section{H. SAMELSON}

Stanford University

Stanford, California

J. P. JANS

University of Washington

Seattle, Washington 98105
J. Dugundji

University of Southern California Los Angeles, California 90007

RICHARD ARENS

University of California

Los Angeles, California 90024

\section{ASSOCIATE EDITORS}
E. F. BECKENBACH
B. H. NeumanN
F. WOLF
K. YosidA

\section{SUPPORTING INSTITUTIONS}

UNIVERSITY OF BRITISH COLUMBIA

CALIFORNIA INSTITUTE OF TECHNOLOGY

UNIVERSITY OF CALIFORNIA

MONTANA STATE UNIVERSITY

UNIVERSITY OF NEVADA

NEW MEXICO STATE UNIVERSITY

OREGON STATE UNIVERSITY

UNIVERSITY OF OREGON

OSAKA UNIVERSITY

UNIVERSITY OF SOUTHERN CALIFORNIA
STANFORD UNIVERSITY

UNIVERSITY OF TOKYO

UNIVERSITY OF UTAH

WASHINGTON STATE UNIVERSITY

UNIVERSITY OF WASHINGTON

AMERICAN MATHEMATICAL SOCIETY CHEVRON RESEARCH CORPORATION TRW SYSTEMS

NAVAL ORDNANCE TEST STATION 


\section{Pacific Journal of Mathematics \\ Vol. 22, No. $1 \quad$ January, 1967}

Charles A. Akemann, Some mapping properties of the group algebras of a

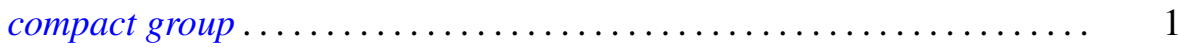

A. V. Boyd, Note on a paper by Uppuluri ..................... 9

Thomas Craig Brown, A semigroup union of disjoint locally finite subsemigroups which is not locally finite .....................

Richard Thomas Bumby and Everett C. Dade, Remark on a problem of

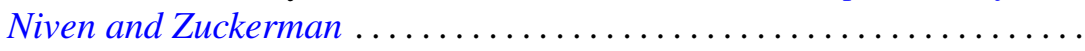

James Calvert, An integral inequality with applications to the Dirichlet problem .........................................

Jack Gary Ceder and Terrance Laverne Pearson, On products of maximally resolvable spaces ....................................

William Guignard Faris, The product formula for semigroups defined by

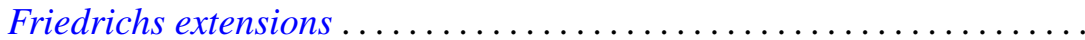

Robert S. Freeman, Closed operators and their adjoints associated with elliptic differential operators ........................ 71

Thomas Lee Hayden, The extension of bilinear functionals ............. 99

Gloria Conyers Hewitt, Limits in certain classes of abstract algebras . . . . . 109

Tilla Weinstein, The dilatation of some standard mappings ........... 117

Mitsuru Nakai, On Evans' kernel ......................... 125

Ernest Levane Roetman, On the biharmonic wave equation ............ 139

Malcolm Jay Sherman, Operators and inner functions ... . .

Walter Laws Smith, On the weak law of large numbers and the generalized elementary renewal theorem

A. J. Ward, On H-equivalence of uniformities: The Isbell-Smith problem 